\title{
The Impact of COVID-19 Pandemic on Lung Cancer Community
}

\author{
Licun $\mathrm{Wu}^{\mathrm{a}, \mathrm{d}}$, Chengke Zhang ${ }^{\mathrm{b}}$, Xiaogang Zhao ${ }^{\mathrm{b}, \mathrm{c}, \mathrm{d}}$
}

\begin{abstract}
Since the outbreak of 2019 novel coronavirus disease (COVID-19) induced by a severe acute respiratory syndrome coronavirus 2 (SARSCoV-2), the pandemic has become a global catastrophe. Patients with cancer especially lung cancer are more vulnerable and susceptible to get infected by the virus SARS-CoV-2. The overwhelming impact of COVID-19 on lung cancer community may result in rise of the incidence and mortality of lung cancer. It would become more obvious in future retrospective studies. Lung cancer patients are believed at higher risk of COVID-19 due to immunosuppression and should be protected by vaccination with priority. Better understanding of SARS-CoV-2 could help develop more effective vaccines to eradicate this disease in the near future.
\end{abstract}

Keywords: COVID-19; SARS-CoV-2; Lung cancer; Immunosuppression

\section{Introduction}

Since the outbreak of 2019 novel coronavirus disease (COVID-19) induced by a severe acute respiratory syndrome coronavirus 2 (SARS-CoV-2), the pandemic has become a global catastrophe resulting in overwhelming impacts on human life

Manuscript submitted February 13, 2021, accepted February 21, 2021

Published online February 24, 2021

a Latner Thoracic Surgery Research Laboratories and Division of Thoracic Surgery, Toronto General Hospital, University Health Network, University of Toronto, Princess Margaret Cancer Research Tower, Toronto, ON, M5G 2C4, Canada

${ }^{b}$ Key Laboratory of Thoracic Cancer, Cheeloo College of Medicine, Shandong University, Ji'nan 250033, Shandong Province, China

'Department of Thoracic Surgery, The Second Hospital, Cheeloo College of Medicine, Shandong University, Ji'nan 250033, Shandong Province, China

${ }^{\mathrm{d} C o r r e s p o n d i n g ~ A u t h o r: ~ L i c u n ~ W u, ~ L a t n e r ~ T h o r a c i c ~ S u r g e r y ~ R e s e a r c h ~ L a b o r a-~}$ tories and Division of Thoracic Surgery, Toronto General Hospital, University Health Network, University of Toronto, Princess Margaret Cancer Research Tower, Toronto, ON, M5G 2C4, Canada. Email: licun.wu@uhnresearch.ca; Xiaogang Zhao, Department of Thoracic Surgery, The Second Hospital, Cheeloo College of Medicine, Shandong University, Ji'nan 250033, Shandong Province, China. Email: zhaoxiaogang@sdu.edu.cn

doi: https://doi.org/10.14740/wjon1367
$[1,2]$. In an effort to prevent the spread of the COVID-19, various activities and academic events including cancer medicare, research and academic conferences have been highly interrupted [3]. Here we specified the multifaceted impacts on lung cancer community.

Patients with cancer have been disproportionately affected by the COVID-19 pandemic including the adverse outcomes in cancer patients with COVID-19, the impact of the COVID-19 pandemic on the delivery of cancer management and the severe disruption to cancer research [4].

With dramatic increase of COVID-19 cases and widespread, the diagnosis and treatment of lung cancer patients have been an increasing challenge due to the overload of public health measures as the lung cancer patients are a highrisk population during the outbreak of COVID-19. The lung cancer patients are badly needed to avoid viral infection. To a great extent, it is difficult to distinguish lung cancer patients from those who had COVID-19 symptoms. This pioneer work pointed out how to apply individual management for lung cancer patients to protect them from COVID-19 [5].

\section{Lung Cancer Patients at High Risk of Infection by SARS-CoV-2}

As recognized, the patients with cancer and COVID-19 were more likely to deteriorate into severe illness than those without cancers [6]. Most strikingly, the patients with lung cancer are more vulnerable and susceptible to get infected by the virus SARS-CoV-2. Considerable evidence has demonstrated that this virus selectively attacks lung tissue [7]. SARS-CoV-2 is interfered primarily by angiotensin-converting enzyme 2 (ACE2) receptor. If the viral load is overwhelming, SARSCoV-2 infection will spread to other organs mainly dependent on ACE2 receptor expression. Cytokine storm and organ crosstalk result in systemic inflammation with multi-organ failure syndromes [8]. The symptoms of lung cancer patients may have some similarity with COVID-19 including fever, cough, shortness of breath and pleural effusion. Majority of them are the elderly, immunosuppressive, accompanying by other complications, leading to them at high risk of COVID-19 prevalence $[9,10]$.

Based on previous studies on the innate and adaptive immune responses elicited by SARS-CoV-2 infection, it has been aware that the immunological pathways likely contribute to severity and survival of COVID-19 patients [11]. Evidence 
showed that high levels of $\mathrm{C}-\mathrm{X}-\mathrm{C}$ motif chemokine ligand 10 (CXCL10), chemokine (C-C motif) ligand 7 (CCL7) and interleukin-1 (IL-1) receptor antagonist in patients with COVID-19 were associated with increased viral load, lung injury, loss of lung function and worse outcome, which may provide better understanding of COVID-19 and deep insights into prognosis and therapeutics [12].

Deep immune profiling of COVID-19 patients published by Mathew et al revealed distinct immunotypes with therapeutic implications using high-dimensional cytometry to analyze 125 COVID-19 patients in comparison with them with recovered and healthy individuals. Three immunotypes were implicated to be capable of helping therapeutic design [13].

\section{Differentially Expressed Genes and Immuno- suppressive Enrichment Networks in Lung Can- cer Patients}

The database was retrieved from database of Gene Expression Profiling Interactive Analysis (GEPIA; http://gepia.cancerpku.cn/). Differential expression analysis was performed to evaluate the differential gene expression profile on chromosomal distribution of gene expression plots across all lung cancer samples and normal tissues (Fig. 1a). Analysis of gene ontology (GO) biological process revealed immunosuppressive pathways involved of down-regulated genes in lung adenocarcinoma patients. The immune-related enrichment networks that the under-expressed genes are highly involved included humoral and adaptive immune response pathways, immune cell adhesion and chemotaxis (Fig. 1b).

All severe COVID-19 patients should be screened for hyperinflammation to identify the subgroup of patients based on their cytokine storm syndromes and immunosuppression. Therapeutic options including steroids, intravenous immunoglobulin, selective cytokine blockade and JAK inhibition could improve the mortality of such patients [14].

\section{The Mortality of Lung Cancer Patients May In- crease Once Infected by SARS-CoV-2}

Cancellation of screening programs and limited outpatient service may lead to delayed diagnosis and shrunk adherence to treatment protocols in lung cancer patients. As a result, both incidence and mortality are expected to increase accordingly [15].

Clinical characteristics, outcomes and risk factors for mortality were studied in patients with cancer and COVID-19 in Hubei, China. Patients with cancer and COVID-19 who were admitted to hospital had a high case-fatality rate. Unfavorable prognostic factors, including receiving chemotherapy within 4 weeks before symptom onset and male sex, might help clinicians to identify patients at high risk of fatal outcomes [16].

There is a retrospective study from a Spanish group reviewing 1,878 medical records of all COVID-19 patients to detect cumulative incidence of COVID-19 in lung cancer patients. They found that lung cancer patients with COVID-19 diagnosis had significantly higher mortality rate than the COVID-19 patients in general population. Dead lung cancer patients were elderly compared to survivors [17]. Cancer is undoubtedly an adverse prognostic indicator on COVID19-related mortality, but what extent effect largely depends on individual patient. Another study showed a population-based analysis of patients hospitalized by COVID-19 with or without cancer. This population-based analysis demonstrates that lung cancer is an independent adverse prognostic factor for those patients with COVID-19 [18]. Sha et al first investigated the impact of COVID-19 on non-COVID-19 lung cancer patients during the pandemic. The study demonstrated the significant impact of the COVID-19 crisis on oncological care, indicating that treatment decisions, follow-up and psycho-oncological support need to be changed during pandemic [19]. A study done by a Korean group analyzed the impact of COVID-19 on lung cancer diagnosis in Korea, where lung cancer incidence continues to rise, and they found that the proportion of patients with advanced non-small-cell lung carcinoma (NSCLC) increased during the COVID-19 pandemic [20].

Treatment disruptions, modifications, or delayed cancer diagnoses due to COVID-19 pandemic may result in clinical consequence for these patients [21]. Since the beginning of the pandemic, many hypotheses have been made regarding anticancer treatments that increase the risk of serious complications of COVID-19. For example, chemotherapy can induce a decrease in neutrophils and lymphocytes, potentially increasing the possibility of infection. Immune checkpoint blockade is a common immunotherapy for lung cancer, but it may increase the risk of inflammatory syndrome and acute COVID-19 respiratory disease. In addition, radiotherapy may also limit bone marrow reserves and cause pneumonia, which will increase complications for COVID-19 patients [22].

However, currently few clinical data support these hypotheses. Researchers from 55 centers in the UK published a prospective observational study of 800 cancer patients and documented COVID-19 infections. About half of the patients $(52 \%)$ had a mild course, $28 \%$ of patients died, and the risk of death was significantly related to similar clinical features reported in other studies: old age, men and complications, such as diabetes and cardiovascular disease [23].

There was no significant correlation between chemotherapy and COVID-19 infection mortality in patients $(281,35 \%)$ who received chemotherapy within 4 weeks after COVID-19, compared with cancer patients who did not receive chemotherapy. In addition, patients receiving immunotherapy, targeted therapy, hormone therapy, surgery or radiotherapy within 4 weeks after surgery have no significant correlation with mortality. Active cancer treatment may not adversely affect the survival rate of cancer patients infected with COVID-19 [24].

COVID-19 has had a significant impact on the prognosis of patients with lung cancer.

The 30-day mortality rate in patients with NSCLC significantly increased from $25 \%$ before COVID-19 to $49 \%$ during COVID-19. For the patients with small-cell lung cancer (SCLC), the 30-day mortality rate jumped from $18 \%$ before COVID-19 to $32 \%$ during COVID-19 [25].

A study from Imperial College London is the largest global analysis of cancer patients with COVID-19 [26]. Such a large 
a The Differentially Expressed Genes On Chromosomes

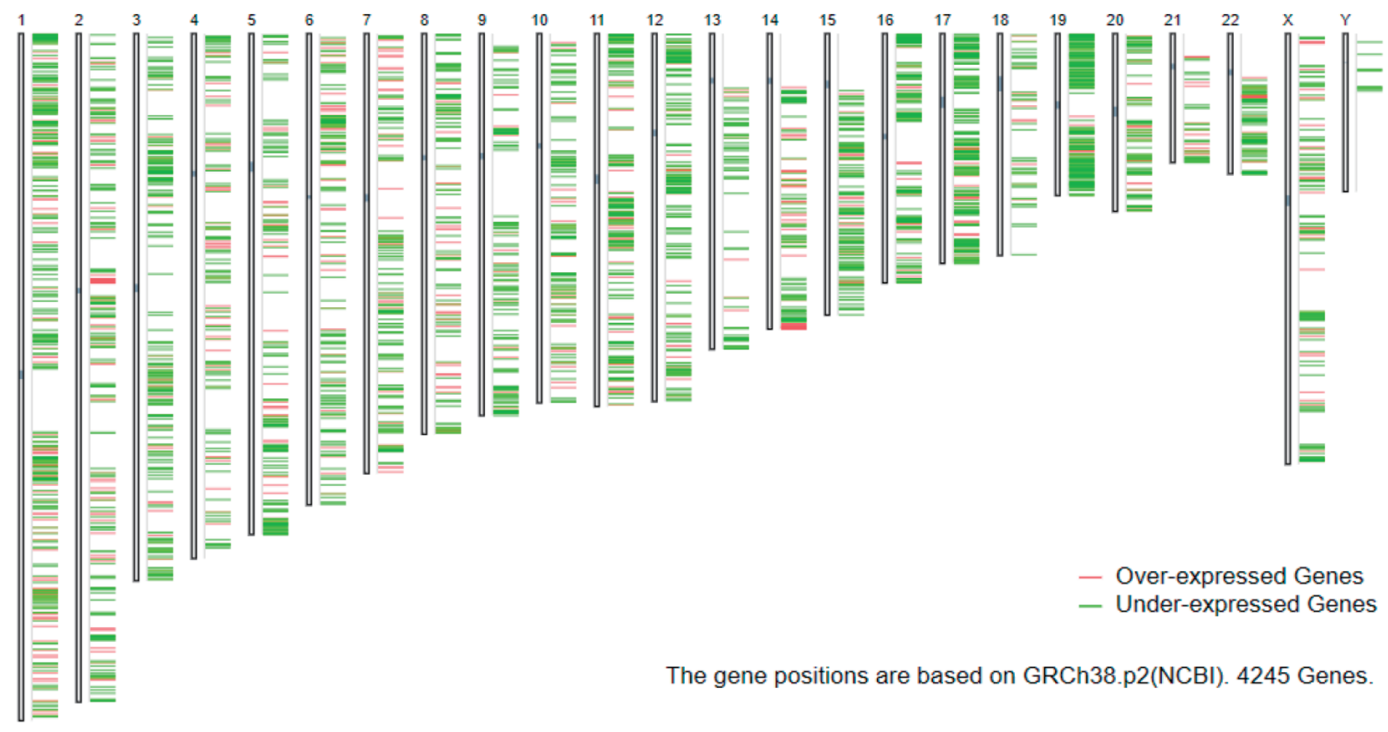

Enrichment network: Gene Ontology biological process of down-regulated genes in lung adenocarcinoma

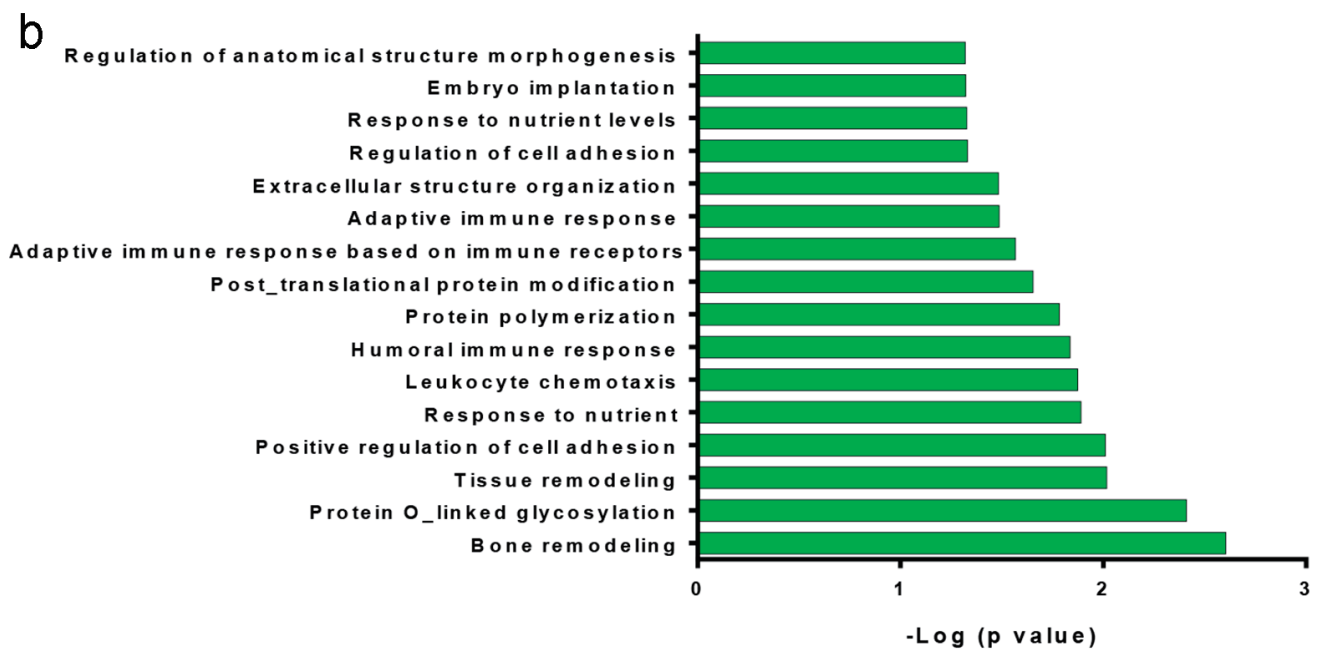

Figure 1. Differentially expressed genes and immunosuppressive enrichment networks in lung cancer patients. The database was achieved from database of Gene Expression Profiling Interactive Analysis (GEPIA; http://gepia.cancer-pku.cn/). Differential expression analysis was performed to evaluate the gene expression profile across all tumor lung cancer samples and normal tissues. Differential method analysis of variance (ANOVA) was used for tumor vs. paired normal samples. (a) Chromosomal distribution of gene expression plots. Default color: over-expressed genes (red); under-expressed genes (green). (b) Gene ontology biological process: immunosuppressive pathways involved of down-regulated genes in lung adenocarcinoma patients.

cohort of cancer patients with COVID-19 showed that these patients are at increased risk of severe outcomes and death. Age, gender and co-existing comorbidities appear to be significant risk factors associated with poorer prognosis. Effectively monitoring and treating this population could help clinicians to minimize the impact of the COVID-19 pandemic on cancer patients and reduce mortality. COVID-19 vaccines would be of the priority to them [27].

To reduce the mortality of lung cancer patients during the
COVID-19 outbreak period, it should be extremely cautious to evaluate the risk of COVID-19 for lung cancer patients [28].

\section{The Impact of COVID-19 Pandemic on Lung Cancer Research}

Systemic search of all publications available from PubMed was performed using "COVID-19/SARS-CoV-2", "lung can- 
a Search from PubMed

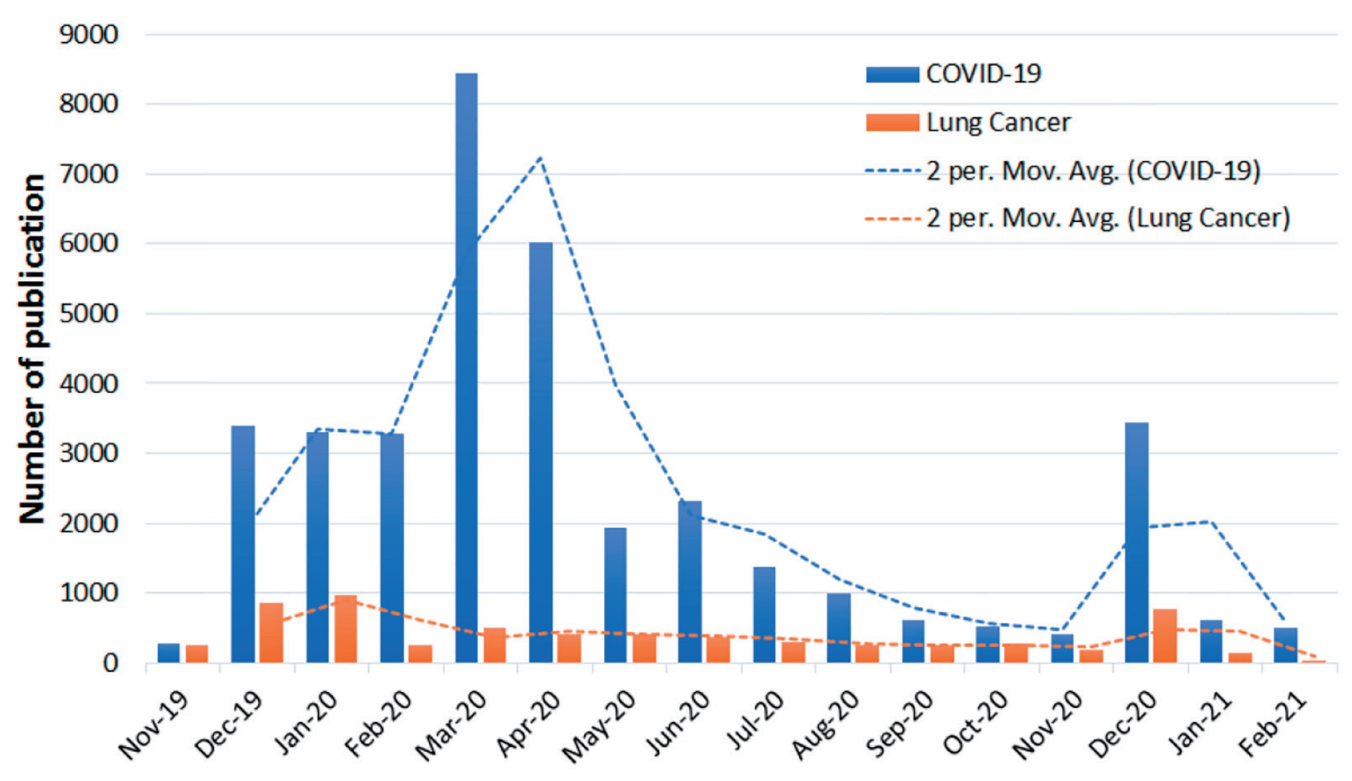

\section{b COVID-19+Lung Cancer}

\section{COVID-19+Lung Cancer}

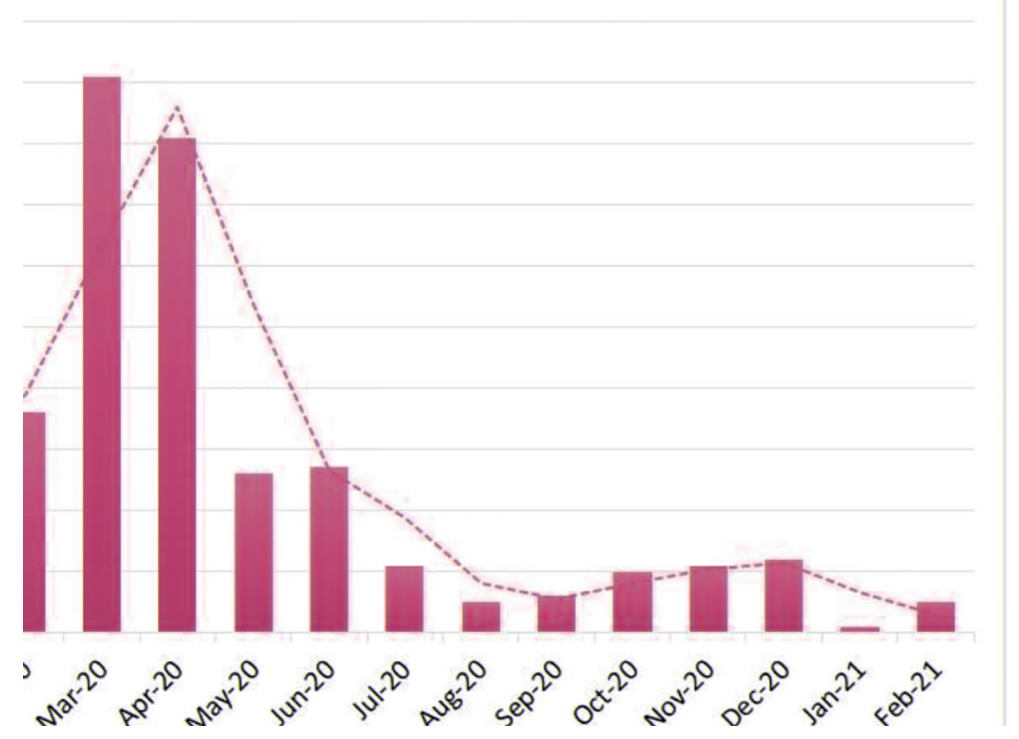

b

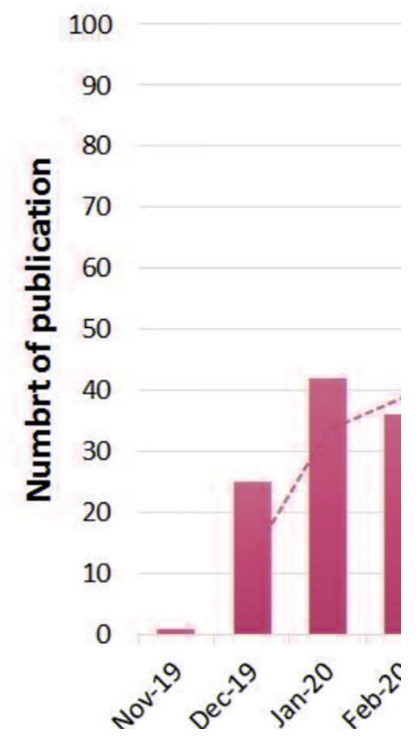

Figure 2. The impact of COVID-19 pandemic on lung cancer research. Published literature was searched from PubMed database between November 2019 and February 10, 2021. All studies on COVID-19, lung cancer and combination of both COVID-19 and lung cancer were presented over time. (a) COVID-19 and lung cancer separately. (b) Studies on both COVID-19 and lung cancer. The trendlines were plotted based on Two Period Moving Average (2 Per. Mov. Aver.).

cer", "COVID-19 and lung cancer". The number of monthly publications from November 2019 to February 10, 2021 was found and plot graphs as a function of number of publications over time (month) are shown in Figure 2. Along with 
the outbreak from the end of 2019, the number of publication increased abruptly from December 2019 to March 2020, and reached a peak in March and April 2020, and maintained at a higher level than the number of publication on lung cancer, while lung studies appeared to maintain flat of the graph (Fig. 2a). Interestingly, the number of studies on lung cancer patients concurrent with COVID-19 seemed to increase at a similar pattern with the studies on COVID-19 alone (Fig. 2b).

At the beginning of the outbreak of such a novel disease, SARS-CoV2 and COVID-19 attracted sufficient curiosity of global medical doctors and research scientists [29-31]. Nowadays, with more understanding of the virus and disease, the number of studies seemed to decrease compared with earlier time. As expected, a variety of vaccines against COVID-19 have been eventually developed and applied for prevention of COVID-19 [32-36]. Hopefully, more effective vaccines could be manufactured to eradicate SARS-CoV-2 transmission and infection in the near future [37].

\section{Concluding Remarks}

In conclusion, the overwhelming impact of COVID-19 on lung cancer community may overexert our expectation, especially the incidence and mortality of lung cancer. It would become more obvious in future retrospective studies. Lung cancer patients are believed at higher risk of COVID-19 due to immunosuppression and should get vaccination with priority. With more profound understanding of SARS-CoV-2, more effective vaccines could be developed to eradicate this disease shortly.

\section{Acknowledgments}

None to declare.

\section{Financial Disclosure}

None to declare.

\section{Conflict of Interest}

There is no conflict of interest.

\section{Author Contributions}

LW contributed to manuscript drafting; LW, CZ and XZ contributed to overall ideas, discussion and review.

\section{Data Availability}

The authors declare that data supporting the findings of this study are available within the article.

\section{References}

1. Zhu N, Zhang D, Wang W, Li X, Yang B, Song J, Zhao X, et al. A novel coronavirus from patients with pneumonia in China, 2019. N Engl J Med. 2020;382(8):727-733.

2. Wang D, Hu B, Hu C, Zhu F, Liu X, Zhang J, Wang B, et al. Clinical characteristics of 138 hospitalized patients with 2019 novel coronavirus-infected pneumonia in Wuhan, China. JAMA. 2020;323(11):1061-1069.

3. Wang C, Horby PW, Hayden FG, Gao GF. A novel coronavirus outbreak of global health concern. Lancet. 2020;395(10223):470-473.

4. Bakouny Z, Hawley JE, Choueiri TK, Peters S, Rini BI, Warner JL, Painter CA. COVID-19 and Cancer: Current Challenges and Perspectives. Cancer Cell. 2020;38(5):629-646.

5. Yang L, Xu HY, Wang Y. [Diagnostic and therapeutic strategies of lung cancer patients during the outbreak of 2019 novel coronavirus disease (COVID-19)]. Zhonghua Zhong Liu Za Zhi. 2020;42(4):292-295.

6. Tian J, Yuan X, Xiao J, Zhong Q, Yang C, Liu B, Cai Y, et al. Clinical characteristics and risk factors associated with COVID-19 disease severity in patients with cancer in Wuhan, China: a multicentre, retrospective, cohort study. Lancet Oncol. 2020;21(7):893-903.

7. Ackermann M, Verleden SE, Kuehnel M, Haverich A, Welte T, Laenger F, Vanstapel A, et al. Pulmonary Vascular Endothelialitis, Thrombosis, and Angiogenesis in Covid-19. N Engl J Med. 2020;383(2):120-128.

8. Loganathan S, Kuppusamy M, Wankhar W, Gurugubelli KR, Mahadevappa VH, Lepcha L, Choudhary AK. Angiotensin-converting enzyme 2 (ACE2): COVID 19 gate way to multiple organ failure syndromes. Respir Physiol Neurobiol. 2021;283:103548.

9. Ledford H. How does COVID-19 kill? Uncertainty is hampering doctors' ability to choose treatments. Nature. 2020;580(7803):311-312.

10. Singh AP, Berman AT, Marmarelis ME, Haas AR, Feigenberg SJ, Braun J, Ciunci CA, et al. Management of lung cancer during the COVID-19 pandemic. JCO Oncol Pract. 2020;16(9):579-586.

11. Vabret N, Britton GJ, Gruber C, Hegde S, Kim J, Kuksin M, Levantovsky R, et al. Immunology of COVID-19: current state of the science. Immunity. 2020;52(6):910941.

12. Vaninov N. In the eye of the COVID-19 cytokine storm. Nat Rev Immunol. 2020;20(5):277.

13. Mathew D, Giles JR, Baxter AE, Oldridge DA, Greenplate $\mathrm{AR}, \mathrm{Wu}$ JE, Alanio $\mathrm{C}$, et al. Deep immune profiling of COVID-19 patients reveals distinct immunotypes with therapeutic implications. Science. 2020;369(6508):eabc8511.

14. Mehta P, McAuley DF, Brown M, Sanchez E, Tattersall RS, Manson JJ, Hlh Across Speciality Collaboration UK. COVID-19: consider cytokine storm syndromes and immunosuppression. Lancet. 2020;395(10229):1033-1034.

15. Lee LYW, Cazier JB, Starkey T, Briggs SEW, Arnold R, Bisht V, Booth S, et al. COVID-19 prevalence and mor- 
tality in patients with cancer and the effect of primary tumour subtype and patient demographics: a prospective cohort study. Lancet Oncol. 2020;21(10):1309-1316.

16. Yang K, Sheng Y, Huang C, Jin Y, Xiong N, Jiang K, Lu $\mathrm{H}$, et al. Clinical characteristics, outcomes, and risk factors for mortality in patients with cancer and COVID-19 in Hubei, China: a multicentre, retrospective, cohort study. Lancet Oncol. 2020;21(7):904-913.

17. Rogado J, Pangua C, Serrano-Montero G, Obispo B, Marino AM, Perez-Perez M, Lopez-Alfonso A, et al. COVID-19 and lung cancer: A greater fatality rate? Lung Cancer. 2020;146:19-22.

18. de Azambuja E, Brandao M, Wildiers H, Laenen A, Aspeslagh S, Fontaine C, Collignon J, et al. Impact of solid cancer on in-hospital mortality overall and among different subgroups of patients with COVID-19: a nationwide, population-based analysis. ESMO Open. 2020;5(5):e000947.

19. Sha Z, Chang K, Mi J, Liang Z, Hu L, Long F, Shi H, et al. The impact of the COVID-19 pandemic on lung cancer patients. Ann Palliat Med. 2020;9(5):3373-3378.

20. Park JY, Lee YJ, Kim T, Lee CY, Kim HI, Kim JH, Park S, et al. Collateral effects of the coronavirus disease 2019 pandemic on lung cancer diagnosis in Korea. BMC Cancer. 2020;20(1):1040.

21. Elkrief A, Kazandjian S, Bouganim N. Changes in lung cancer treatment as a result of the coronavirus disease 2019 pandemic. JAMA Oncol. 2020.

22. van de Haar J, Hoes LR, Coles CE, Seamon K, Frohling $\mathrm{S}$, Jager D, Valenza F, et al. Caring for patients with cancer in the COVID-19 era. Nat Med. 2020;26(5):665-671.

23. Lee LY, Cazier JB, Angelis V, Arnold R, Bisht V, Campton NA, Chackathayil J, et al. COVID-19 mortality in patients with cancer on chemotherapy or other anticancer treatments: a prospective cohort study. Lancet. 2020;395(10241):1919-1926.

24. Disis ML. Oncology and COVID-19. JAMA. 2020;324(12):1141-1142.

25. Reyes R, Lopez-Castro R, Auclin E, et al. Impact of COVID-19 pandemic in the diagnosis and prognosis of lung cancer. Presented at: 2020 World Conference on
Lung Cancer Singapore; January 28-31; Virtual. Abstract 3700.

26. Zhang H, Han H, He T, Labbe KE, Hernandez AV, Chen $\mathrm{H}$, Velcheti $\mathrm{V}$, et al. Clinical characteristics and outcomes of COVID-19-infected cancer patients: a systematic review and meta-analysis. J Natl Cancer Inst. 2020.

27. Dai M, Liu D, Liu M, Zhou F, Li G, Chen Z, Zhang Z, et al. Patients with Cancer Appear More Vulnerable to SARS-CoV-2: A Multicenter Study during the COVID-19 Outbreak. Cancer Discov. 2020;10(6):783-791.

28. Xu Y, Liu H, Hu K, Wang M. [Clinical Management of Lung Cancer Patients during the Outbreak of 2019 Novel Coronavirus Disease (COVID-19)]. Zhongguo Fei Ai Za Zhi. 2020;23(3):136-141.

29. Rasmussen AL. On the origins of SARS-CoV-2. Nat Med. 2021;27(1):9.

30. Norton A, Mphahlele J, Yazdanpanah Y, Piot P, Bayona MT. Strengthening the global effort on COVID-19 research. Lancet. 2020;396(10248):375.

31. Yan R, Zhang Y, Li Y, Xia L, Guo Y, Zhou Q. Structural basis for the recognition of SARS-CoV-2 by full-length human ACE2. Science. 2020;367(6485):1444-1448.

32. Polack FP, Thomas SJ, Kitchin N, Absalon J, Gurtman A, Lockhart S, Perez JL, et al. Safety and efficacy of the BNT162b2 mRNA COVID-19 vaccine. N Engl J Med. 2020;383(27):2603-2615.

33. Walsh EE, Frenck RW, Jr., Falsey AR, Kitchin N, Absalon J, Gurtman A, Lockhart S, et al. Safety and immunogenicity of two RNA-based COVID-19 vaccine candidates. N Engl J Med. 2020;383(25):2439-2450.

34. Haynes BF, Corey L, Fernandes P, Gilbert PB, Hotez PJ, Rao S, Santos MR, et al. Prospects for a safe COVID-19 vaccine. Sci Transl Med. 2020;12(568):abe0948.

35. Heaton PM. The COVID-19 vaccine-development multiverse. N Engl J Med. 2020;383(20):1986-1988.

36. Knoll MD, Wonodi C. Oxford-AstraZeneca COVID-19 vaccine efficacy. Lancet. 2021;397(10269):72-74.

37. Le TT, Cramer JP, Chen R, Mayhew S. Evolution of the COVID-19 vaccine development landscape. Nat Rev Drug Discov. 2020;19(10):667-668. 